\title{
RECURSIVELY ENUMERABLE SETS OF POSITIVE INTEGERS AND THEIR DECISION PROBLEMS
}

\author{
EMIL L. POST
}

Introduction. Recent developments of symbolic logic have considerable importance for mathematics both with respect to its philosophy and practice. That mathematicians generally are oblivious to the importance of this work of Gödel, Church, Turing, Kleene, Rosser and others as it affects the subject of their own interest is in part due to the forbidding, diverse and alien formalisms in which this work is embodied. Yet, without such formalism, this pioneering work would lose most of its cogency. But apart from the question of importance, these formalisms bring to mathematics a new and precise mathematical concept, that of the general recursive function of Herbrand-GödelKleene, or its proved equivalents in the developments of Church and Turing. ${ }^{1}$ It is the purpose of this lecture to demonstrate by example that this concept admits of development into a mathematical theory much as the group concept has been developed into a theory of groups. Moreover, that stripped of its formalism, such a theory admits of an intuitive development which can be followed, if not indeed pursued, by a mathematician, layman though he be in this formal field. It is this intuitive development of a very limited portion of a sub-theory of the hoped for general theory that we present in this lecture. We must emphasize that, with a few exceptions explicitly so noted, we have obtained formal proofs of all the consequently mathematical theorems here developed informally. Yet the real mathematics involved must lie in the informal development. For in every instance the informal "proof" was first obtained; and once gotten, transforming it into the formal proof turned out to be a routine chore. ${ }^{2}$

We shall not here reproduce the formal definition of recursive function of positive integers. A simple example of such a function is an

An address presented before the New York meeting of the Society on February 26, 1944, by invitation of the Program Committee; received by the editors March 25, 1944.

${ }^{1}$ For "general recursive function" see [9] ([8] a prerequisite), [12] and [11]; for Church's " $\lambda$-defineability," [1] and [6]; for Turing's "computability," [24] and the writer's related [18]. To this may be added the writer's method of "canonical systems and normal sets" [19]. See pp. 39-42 and bibliography of [6] for a survey of the literature and further references. Numbers in brackets refer to the bibliography at the end of the paper.

2 Our present formal proofs, while complete, will require drastic systematization and condensation prior to publication. 
arbitrary polynomial $P\left(x_{1}, x_{2}, \cdots, x_{n}\right)$, with say non-negative integral coefficients, and not identically zero. If the $x$ 's are assigned arbitrary positive integral values expressed, for example, in the arabic notation, the algorithms for addition and multiplication in that notation enable us to calculate the corresponding positive integral value of the polynomial. That is, $P\left(x_{1}, x_{2}, \cdots, x_{n}\right)$ is an effectively calculable function of positive integers. The importance of the technical concept recursive function derives from the overwhelming evidence that it is coextensive with the intuitive concept effectively calculable function. ${ }^{8}$

A set of positive integers is said to be recursively enumerable if there is a recursive function $f(x)$ of one positive integral variable whose values, for positive integral values of $x$, constitute the given set. The sequence $f(1), f(2), f(3), \cdots$ is then said to be a recursive enumeration of the set. The corresponding intuitive concept is that of an effectively enumerable set of positive integers. To prepare us in part for our intuitive approach, consider the following three examples of recursively enumerable sets of positive integers.
(a):
(b):
(c):

$1^{2}, 2^{2}, 3^{2}, \cdots$.

$1,2,2^{1+2}, 2^{1+2+2^{1+2}}, \cdots$.

$1^{2}, 2^{2}, 3^{2}, \ldots$

$1^{3}, 2^{3}, 3^{3}, \ldots$

$1^{4}, 2^{4}, 3^{4}, \ldots$

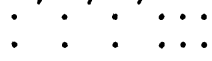

In the first example, the set is given by a recursive enumeration thereof via the recursive function $x^{2}$. In the second example, the set is generated in a linear sequence, each new element being effectively obtained from the elements previously generated, in this case by raising 2 to the power the sum of the preceding elements. The set is effectively enumerable, since the $n$th element of the sequence can be found, given $n$, by regenerating the sequence through its first $n$ elements. In the third example, we rather imagine the positive integers $1,2,3, \ldots$ generated in their natural order, and, as each positive integer $n$ is generated, a corresponding process set up which generates $n^{2}, n^{3}, n^{4}, \cdots$, all these to be in the set. Actually, the standard method for proving that an enumerable set of enumerable sets is enumerable yields an effective enumeration of the set.

\footnotetext{
${ }^{8}$ See Kleene $[13$, footnote 2$]$. In the present paper, "recursive function" means "general recursive function."
} 
Several more examples would have to be given to convey the writer's concept of a generated set, in the present instance of positive integers. Suffice it to say that each element of the set is at some time written down, and earmarked as belonging to the set, as a result of predetermined effective processes. It is understood that once an element is placed in the set, it stays there. The writer elsewhere has referred to a generalization which may be restated every generated set of positive integers is recursively enumerable. ${ }^{4}$ For comparison purposes this may be resolved into the two statements: every generated set is effectively enumerable, every effectively enumerable set of positive integers is recursively enumerable. The first of these statements is applicable to generated sets of arbitrary symbolic expressions; their converses are immediately seen to be true. We shall find the above concept and generalization very useful in our intuitive development. But while we shall frequently say, explicitly or implicitly, "set so and so of positive integers is a generated, and hence recursively enumerable set," as far as the present enterprise is concerned that is merely to mean "the set has intuitively been shown to be a generated set; it can indeed be proved to be recursively enumerable." Likewise for other identifications of informal concepts with corresponding mathematically defined formal concepts.

At a few points in our informal development we have to lean upon the formal development. The latter is actually yet another formalism, due to the writer [19] but proved completely equivalent to that of general recursive function. It will suffice to give the equivalent of "recursively enumerable set of positive integers" in this development.

A positive integer $n$ is represented in the most primitive fashion by a succession $11 \cdots 1$ of $n$ strokes. For working purposes, we introduce the letter $b$, and consider "strings" of 1 's and $b$ 's such as $11 b 1 b b 1$. An operation on such strings such as " $b 1 b P$ produces $P 1 b b 1$ " we term a normal operation. This particular normal operation is applicable only to strings starting with $b 1 b$, and the derived string is then obtained from the given string by first removing the initial $b 1 b$, and then tacking on $1 b b 1$ at the end. Thus $b 1 b b$ becomes $b 1 b b 1$. " $g P$ produces $P \mathrm{~g}^{\prime \prime}$ " is the form of an arbitrary normal operation. A system in normal form, or normal system, is given by an initial string $A$ of 1 's and $b$ 's, and a finite set of normal operations " $g_{i} P$ produces $P g_{i}^{\prime}$," $i=1,2, \cdots, \mu$. The derived strings of the system are $A$ and all strings obtainable from $A$ be repeated applications of the $\mu$ normal

' See [19, p. 201 and footnote 18]. In this connection note Kleene's use of the word "Thesis" in [14, p. 60]. We still feel that, ultimately, "Law" will best describe the situation [18]. 
operations. Each normal system uniquely defines a set, possibly null, of positive integers, namely the integers represented by those derived strings which are strings of 1 's only. It can then be proved that every recursively enumerable set of positive integers is the set of positive integers defined by some normal system, and conversely. ${ }^{5}$ We here, as below, arbitrarily extend the concept recursively enumerable set to include the null set.

By the basis $B$ of a normal system, and of the recursively enumerable set of positive integers it defines, we mean the string of letters and symbols here represented by

$$
A ; g_{1} P \text { produces } P g_{1}^{\prime}, \cdots, g_{\mu} P \text { produces } P g_{\mu}^{\prime} \text {. }
$$

When meaningfully interpreted, $B$ determines the normal system, and recursively enumerable set of positive integers, in question. Each basis is but a finite sequence of the symbols $1, b, P$, the comma, semicolon and the letters of the word "produces." The set of bases is therefore enumerably infinite, and can indeed be effectively generated in a sequence of distinct elements

$$
O: \quad B_{1}, B_{2}, B_{3}, \cdots .
$$

Since each $B_{\boldsymbol{i}}$ defines a unique recursively enumerable set of positive integers and each such set is defined by at least one $B_{i}, O$ is also an ordering of all recursively enumerable sets of positive integers, though each set will indeed recur an infinite number of times in $O$. We may then say, in classical terms, that whereas there are $2^{\mathbf{s}_{0}}$ arbitrary sets of positive integers, there are but $\boldsymbol{\aleph}_{0}$ recursively enumerable sets.

By the decision problem of a given set of positive integers we mean the problem of effectively determining for an arbitrarily given positive integer whether it is, or is not, in the set. While, in a certain sense, the theory of recursively enumerable sets of positive integers is potentially as wide as the theory of general recursive functions, the decision problems for such sets constitute a very special class of decision problems. Nevertheless they are important, as is shown by the following special and general examples.

One of the problems posed by Hilbert in his Paris address of 1900 [10, problem 10] is the problem of determining for an arbitrary diophantine equation with rational integral coefficients whether it has, or has not, a solution in rational integers. If the variables in a

${ }^{5}$ We have thus restricted the normal operations and normal systems of [19] because of the following result. If in the initial string and in the normal operations of a normal system with primitive letters $1, a_{1}^{\prime}, \cdots, a_{\mu^{\prime}}^{\prime}$, each $a_{i}^{\prime}, i=1, \cdots, \mu^{\prime}$, is replaced by $b 1 \cdots 1 b$ with $i$ 1's, a normal system with primitive letters $1, b$ results, defining the same set of strings on 1 only as the original normal system. 
diophantine equation be chosen from a given enumerably infinite set of variables, it is clear that the set of diophantine equations is enumerably infinite. Indeed they can be effectively put into one-one correspondence with the set of positive integers. Since for any one diophantine equation, and assignment of rational integral values to its variables, it can be effectively determined whether or no the equation is satisfied by those values, the set of diophantine equations having rational integral solutions can be generated. The corresponding integers under the above one-one correspondence can then also be generated, and, indeed, constitute a recursively enumerable set of positive integers. ${ }^{6}$ And under that correspondence, Hilbert's problem is transformed into the decision problem of that recursively enumerable set.

The assertions of an arbitrary symbolic $\operatorname{logic}^{7}$ constitute a generated set $A$ of what may be called symbol-complexes or formulas. We assume that $A$ is a subset of an infinite generated set $E$ of symbolcomplexes, which in one case may be the set of meaningful enunciations of the logic, in another the set of all symbol-complexes of a given mode of symbolization. The decision problem of the logic, more precisely its deducibility problem [3], is then the problem of determining of an arbitrary member of $E$ whether it is, or is not, in $A$. Granting that every generated set is effectively enumerable, the members of $E$ can be effectively set in one-one correspondence with the set of positive integers. The positive integers corresponding to the members of $A$ then constitute a generated, and hence, under our generalization, a recursively enumerable set of positive integers. And under that correspondence the decision problem of the symbolic logic is transformed into the decision problem of this recursively enumerable set of positive integers.

Closely related to the technical concept recursively enumerable set of positive integers is that of a recursive set of positive integers. This is a set for which there is a recursive function $f(x)$ such that $f(x)$ is say 2 when $x$ is a positive integer in the set, 1 when $x$ is a positive integer not in the set. We may also make this the definition of the decision problem of the set being recursively solvable. For 2 and 1 may be regarded as the two possible truth-values, true, false, of the proposition "positive integer $x$ is in the set," and the definition of recursive set is equivalent to this truth-value being recursively calculable for all positive integers $x$. If then recursive function is coextensive with

- In view of [17] we inadvertantly carried through our formal verification with "rational integral solution" replaced by "positive integral solution."

${ }^{7}$ See Church [5, p. 225] for our omitting the qualifying "finitary." 
effective calculability, recursive solvability is coextensive with solvability in the intuitive sense. In particular, the decision problem of a recursively enumerable set would be solvable or unsolvable according as the set is, or is not, recursive. More generally than in our two illustrations, through the more precise mechanism of Gödel representations [8], a wide variety of decision and other problems are transformed into problems about positive integers; and whether those problems are, or are not, solvable in the intuitive sense would be equivajent to their being, or not being, recursively solvable in the precise te ${ }_{c}$ hnical sense.

Gödel's classic theorem on the incompleteness and extendibility of symbolic 1 ogics [8] in all but wording led him to the recursive unsolvability of a generalization of the above problem of Hilbert $[8,9$, 22]. Church explicitly formulated the concept of recursive unsolvability, and arrived at the unsolvability of a number of problems; certainly he proved them recursively unsolvable [1-4]. The above problem of Hilbert begs for an unsolvability proof (see [17]). Like the classic unsolvability proofs, these proofs are of unsolvability by means of given instruments. What is new is that in the present case these instruments, in effect, seem to be the only instruments at man's disposal.

Related to the question of solvability or unsolvability of problems is that of the reducibility or non-reducibility of one problem to another. Thus, if problem $P_{1}$ has been reduced to problem $P_{2}$, a solution of $P_{2}$ immediately yields a solution of $P_{1}$, while if $P_{1}$ is proved to be unsolvable, $P_{2}$ must also be unsolvable. For unsolvable problems the concept of reducibility leads to the concept of degree of unsolvability, two unsolvable problems being of the same degree of unsolvability if each is reducible to the other, one of lower degree of unsolvability than another if it is reducible to the other, but that other is not reducible to it, of incomparable degrees of unsolvability if neither is reducible to the other. A primary problem in the theory of recursively enumerable sets is the problem of determining the degrees of unsolvability of the unsolvable decision problems thereof. We shall early see that for such problems there is certainly a highest degree of unsolvability. Our whole development largely centers on the single question of whether there is, among these problems, a lower degree of unsolvability than that, or whether they are all of the same degree of unsolvability. Now in his paper on ordinal logics [26, section 4], Turing presents as a side issue a formulation which can immediately be restated as the general formulation of the "recursive reducibility" of one problem to another, and proves a result which immediately 
generalizes to the result that for any "recursively given" unsolvable problem there is another of higher degree of unsolvability. ${ }^{8}$ While his theorem does not help us in our search for that lower degree of unsolvability, his formulation makes our problem precise. It remains a problem at the end of this paper. But on the way we do obtain a number of special results, and towards the end obtain some idea of the difficulties of the general problem.

1. Recursive versus recursively enumerable sets. The relationship between these two concepts is revealed by the following

THEOREM. $A$ set of positive integers is recursive when and only when both it and its complement with respect to the set of all positive integers are recursively enumerable. ${ }^{\circ}$

For simplicity, we assume both the set $S$ and its complement $\bar{S}$ to be infinite. If, then, $S$ is recursive, there is an effective method for telling of any positive integer $n$ whether it is, or is not, in $S$. Generate the positive integers $1,2,3, \cdots$ in their natural order, and, as a positive integer is generated, test its being or not being in $S$. Each time a positive integer is thus found to be in $S$, write it down as belonging to $S$. Thus, an effective process is set up for effectively enumerating the elements of $S$. Hence, $S$ is recursively enumerable. Likewise $\bar{S}$ can be shown to be recursively enumerable.

Conversely, let both $S$ and $\bar{S}$ be recursively enumerable, and let $n_{1}, n_{2}, n_{3}, \cdots$ be a recursive enumeration of $S ; m_{1}, m_{2}, m_{3}, \cdots$, of $S$. Given a positive integer $n$, generate in order $n_{1}, m_{1}, n_{2}, m_{2}, n_{3}, m_{3}$, and so on, comparing each with $n$. Since $n$ must be either in $S$ or in $\bar{S}$, in a finite number of steps we shall thus come across an $n_{i}$ or $m_{j}$ identical with $n$, and accordingly discover $n$ to be in $S$, or $\bar{S}$. An effective method is thus set up for determining of any positive integer $n$ whether it is, or is not, in $S$. Hence, $S$ is recursive.

CoROLLARY. The decision problem of a recursively enumerable set is recursively solvable when and only when its complement is recursively enumerable.

For then and only then is the recursively enumerable set recursive.

It is readily proved that the logical sum and logical product of two

${ }^{8}$ Both our generalization of his formulation and of his theorem have been carried through, rather hastily, by the formalism of [19], without, as yet, an actual equivalence proof. It may be that Tarski's Theorem 9.1 [23] can be transformed into a like absolute theorem.

- The only portion of this theorem we can find in the literature is Rosser's Corollary II $[20$, p. 88]. 
recursively enumerable sets are recursively enumerable, the complement of a recursive set, and the logical sum, and hence logical product, of two recursive sets are recursive.

Clearly, any finite set of positive integers is recursive. For if $n_{1}, n_{2}, \cdots, n_{\nu}$ are the integers in question, we can test $n$ being, or not being, in the set by directly comparing it with $n_{1}, n_{2}, \cdots, n_{\nu}{ }^{10}$ Likewise for a set whose complement is finite. For arbitrary infinite sets we have the following result of Kleene [12]. An infinite set of positive integers is recursive when and only when it admits of a recursive enumeration without repetitions in order of magnitude. Indeed, if $n_{1}, n_{2}, n_{3}, \ldots$ is a recursive enumeration of $S$ without repetitions in order of magnitude, all $n_{i}$ 's beyond the $n$th must exceed $n$. Hence we can test $n$ being, or not being, in $S$ by generating the first $n$ members of the given recursive enumeration of $S$, and seeing whether $n$ is, or is not, one of them. Conversely, if infinite $S$ is recursive, the recursive enumeration thereof we set up in the proof of our first theorem is of the elements of $S$ without repetition, and in order of magnitude.

A direct consequence of the first half of the last result is the following

THEOREM. Every infinite recursively enumerable set contains an infinite recursive set.

For, if $n_{1}, n_{2}, n_{3}, \ldots$ is a recursive enumeration of an infinite set $S$, for each $n_{i}$ there must be, in this sequence, a later $n_{j}>n_{i}$. Hence, generate the elements $n_{1}, n_{2}, n_{3}, \cdots$ in order, and let $m_{1}=n_{1}, m_{2}=n_{i_{2}}$, the first $n_{i}$ greater than $n_{1}, m_{3}=n_{i_{3}}$, the first $n_{i}$ beyond $n_{i_{2}}$ greater than $n_{i_{2}}$, and so on. The sequence $m_{1}, m_{2}, m_{3}, \cdots$ is then a recursive enumeration of a subset of $S$ without repetitions in order of magnitude. That subset is therefore infinite, and recursive.

Basic to the entire theory is the following result we must credit to Church, Rosser, Kleene, jointly $[1,20,12]$.

THEOREM. There exists a recursively enumerable set of positive integers which is not recursive. ${ }^{11}$

By our first theorem this is equivalent to the existence of a recursively enumerable set of positive integers whose complement is

${ }^{10}$ The mere existence of a general recursive function defining the finite set is in question. Whether, given some definition of the set, we can actually discover what the members thereof are, is a question for a theory of proof rather than for the present theory of finite processes. For sets of finite sets the situation is otherwise, as seen in $\$ 11$.

II In each of our existence theorems we show how to set up the basis of the set in question-at least, the corresponding formal proof does exactly that. 
not recursively enumerable. Generate in order the distinct bases $B_{1}, B_{2}, B_{3}, \cdots$ of all recursively enumerable sets of positive integers as mentioned in the introduction, and keep track of these bases as the first, second, third, and so on, in this enumeration $O$. As the $n$th basis $B_{n}$ is generated, with $n=1,2,3, \cdots$, set going the processes whereby the corresponding recursively enumerable set is generated, and whenever $n$ is thus generated by $B_{n}$, place $n$ in a set $U$. Being a generated set of positive integers, $U$ is recursively enumerable. A positive integer $n$, then, is, or is not, in $U$ according as it is, or is not, in the $n$th recursively enumerable set in $O$ considered as an ordering of all recursively enumerable sets. Hence, $n$ is, or is not, in $\bar{U}$, the complement of $U$, according as it is not, or is, in the $n$th set in $O$. We thus see that $\bar{U}$ differs from each recursively enumerable set in the presence or absence of at least one positive integer. Hence $\bar{U}$ is not recursively enumerable.

CoROLlary. There exists a recursively enumerable set of positive integers whose decision problem is recursively unsolvable.

Taken singly, finite sets, or sets whose complements are finite, are rather trivial examples of recursive sets. On the other hand, if we define two sets of positive integers to be abstractly the same if one can be transformed into the other by a recursive one-one transformation of the set of all positive integers into itself, then all infinite recursive sets with infinite complements are abstractly the same. Our theory being essentially an abstract theory of recursively enumerable sets, our interest therefore centers in recursively enumerable sets that are not recursive. Such sets, as well as their complements, are always infinite. We do not further pursue the question of two sets being abstractly the same, for that is but a special case of each set being oneone reducible to the other $(\$ 4)$.

2. A form of Gödel's theorem. Given any basis $B$, and positive integer $n$, the couple $(B, n)$ may be used to represent the proposition, true or false, " $n$ is in the set generated by $B$." By interlacing the process for generating the distinct bases in the sequence $B_{1}, B_{2}, B_{3}, \cdots$ and the process for generating the positive integers in the sequence $1,2,3, \cdots$ by the addition of 1 's, we can effectively generate the distinct couples $(B, n)$ in the single infinite sequence

$$
O^{\prime}: \quad\left(B_{1}, 1\right),\left(B_{2}, 1\right),\left(B_{1}, 2\right),\left(B_{3}, 1\right),\left(B_{2}, 2\right),\left(B_{1}, 3\right), \ldots .
$$

On the one hand, the set of all couples $(B, n)$ is thus a generated set of expressions which we shall call $E$. On the other hand, $O^{\prime}$ leads to an effective 1-1 correspondence between the members of $E$ and the 
set of positive integers, $(B, n)$ corresponding to $m$ if $(B, n)$ is the $m$ th member of $O^{\prime}$. We may call $m$ the Gödel representation ${ }^{12}$ of $(B, n)$. Given a generated subset of $E$, the Gödel representations of its members will constitute a generated set of positive integers, and conversely. Thus, in the former case we can generate the members of the subset of $E$, and, as a couple $(B, n)$ is generated, find its Gödel representation $m$ by regenerating $O^{\prime}$. The set of these $m$ 's is thus a generated set. Likewise for the converse. If, therefore, we formally define a subset of $E$ to be recursively enumerable if the set of Gödel representations of its members is recursively enumerable, ${ }^{18}$ we can conclude that every generated subset of $E$ is recursively enumerable, and, of course, conversely. Similarly for a like formal definition of a recursive subset of $E$.

While $E$ is just the set of couples $(B, n)$, it may be interpreted as the set of enunciations " $n$ is in the set generated by $B$. " The subset $T$ of $E$ consisting of those couples $(B, n)$ for which $n$ is in the set generated by $B$ may then be interpreted as the set of true propositions in $E$, while $\bar{T}$, the complement of $T$ with respect to $E$, consists of the false propositions in $E$.

Actually, $T$ itself can be generated as follows. Generate $B_{1}, B_{2}$, $B_{3}, \cdots$ in order. As a $B$ is generated, set up the process for generating the set of positive integers determined by $B$, and, whenever a positive integer $n$ is thus generated, write down the couple $(B, n)$. Each $(B, n)$ for which $n$ is in the set generated by $B$ will thus be written down, and conversely. This generated set of $(B, n)$ 's is then $T$. We therefore conclude that $T$ is recursively enumerable.

Now let $F$ be any recursively enumerable subset of $\bar{T}$. If $(B, n)$ is in $F$, it is in $\bar{T}$, and hence $n$ is certainly not in the set generated by $B$. Now generate the members of $F$, and if $(B, n)$ is thus generated, find the $n$th member $B_{n}$ of $O: B_{1}, B_{2}, B_{3}, \cdots$, and if $B_{n}$ is $B$, place $n$ in a set of positive integers $S_{0}$. Since $S_{0}$ is thus a generated set of positive integers, it is recursively enumerable. It will therefore be determined by some basis $B$. Let this basis be in the $\nu$ th in $O$, that is, let the basis be $B_{v}$, and form the couple $\left(B_{v}, \nu\right)$. Now by construction, $S_{0}$ consists of those members of $F$ of the form $\left(B_{n}, n\right)$. Suppose that $\left(B_{v}, \nu\right)$ is in $F$. Then, on the one hand, proposition $\left(B_{v}, \nu\right)$ being false,

12 Rather is the Gödel representation in [8] not just an effectively corresponding positive integer, but one which, when expressed according to a specific algorithm, is "formally similar," in the sense of Ducasse [7, p. 51], to the symbolic expression represented.

${ }^{13}$ In our own development [19], "recursively enumerable subset of $E^{\text {" is defined }}$ directly as a normal subset of $E$, or rather of the set of symbolic representations of the members of $E$. 
$\nu$ would not be in the set generated by $B_{\nu}$, that is (1): $\nu$ would not be in $S_{0}$. But $\left(B_{v}, \nu\right)$ being of the form $\left(B_{n}, n\right),(2): \nu$ would be in $S_{0}$. Our assumption thus leading to a contradiction, it follows that $\left(B_{v}, \nu\right)$ is not in $F$. But $\nu$ can only be in $S_{0}$ by $\left(B_{\nu}, \nu\right)$ being in $F$. Hence, $\nu$ is not in $S_{0}$. Finally, $\left(B_{\nu}, \nu\right)$ as proposition says that $\nu$ is in $S_{0}$. The proposition $\left(B_{\nu}, \nu\right)$ is therefore false, that is $\left(B_{\nu}, \nu\right)$ is in $\bar{T}$.

For any recursively enumerable subset $F$ of $\bar{T}$ there is then always this couple $\left(B_{\nu}, \nu\right)$ in $\bar{T}$, but not in $F$. On the one hand, then, $\bar{T}$ can never be $F$. Hence, $\bar{T}$ is not recursively enumerable. By the definitions of this section, and the first theorem of the last, it follows that $T$, while recursively enumerable, is not recursive. By the decision problem of $T$ we mean the problem of determining for an arbitrarily given member of $E$ whether it is, or is not, in $T$. But that can be interpreted as the decision problem for the class of recursively enumerable sets of positive integers, that is, the problem of determining for any arbitrarily given recursively enumerable set, that is, arbitrarily given basis $B$ of such a set, and arbitrary positive integer $n$ whether $n$ is, or is not, in the set generated by $B$. We may therefore say that the decision problem for the class of all recursively enumerable sets of positive integers is recursively unsolvable, and hence, in all probability, unsolvable in the intuitive sense.

On the other hand, since $\left(B_{\nu}, \nu\right)$ of $\bar{T}$ is not in $F, T$ and $F$ together can never exhaust $E$. Now $T$, or any recursively enumerable subset $T^{\prime}$ of $T$, in conjunction with $F$ may be called a recursively generated logic relative to the class of enunciations $E$. For the appearance of $(B, n)$ in $T^{\prime}$ assures us of the truth of the proposition " $n$ is in the set generated by $B$," while its presence in $F$ would guarantee its falseness. We can then say that no recursively generated logic relative to $E$ is complete, since $F$ alone will lead to the $\left(B_{v}, \nu\right)$ which is neither in $T^{\prime}$ nor in $F$. That is, $\left(B_{v}, \nu\right)$ is undecidable in this logic. Moreover, if, with a given "basis" for $F$, the above argument is carried through formally, ${ }^{14}$ the recursively enumerable $S_{0}$ obtained above will actually be given by a specific basis $B$ which can be constructed by that formal argument. Having found this $B$, we can then regenerate $O: B_{1}, B_{2}, B_{3}, \cdots$, until $B$ is reached, and thus determine the $\nu$ such that $B=B_{\nu}$. That is, given the basis of $F$, the $\left(B_{v}, \nu\right)$ in $\bar{T}$ and not in $F$ can actually be found. If then we add this $\left(B_{v}, \nu\right)$ to $F$, a wider recursively enumerable subset $F^{\prime}$ of $\bar{T}$ results. We may then say that every recursively generated logic relative to $E$ can be extended. Outwardly, these two results, when formally developed, seem to be

${ }^{14}$ Here, the basis of $F$ may be taken to be the basis of the recursively enumerable set of Gödel representations of the members of $F$. But see the preceding footnote. 
Gödel's theorem in miniature. But in view of the generality of the technical concept general recursive function, they implicitly, in all probability, justify the generalization that every symbolic logic is incomplete and extendible relative to the class of propositions constituting $E .^{15}$ The conclusion is unescapable that even for such a fixed, well defined body of mathematical propositions, mathematical thinking is, and must remain, essentially creative. To the writer's mind, this conclusion must inevitably result in at least a partial reversal of the entire axiomatic trend of the late nineteenth and early twentieth centuries, with a return to meaning and truth as being of the essence of mathematics.

3. The complete set $K$; creative sets. Return now to the effective 1-1 correspondence between the set $E$ of distinct $(B, n)$ 's and the set of positive integers obtained via the effective enumeration $O^{\prime}$ of $E$. Since $T$ is a recursively enumerable subset of $E$, the positive integers corresponding to the elements of $T$ constitute a recursively enumerable set of positive integers, $K$. We shall call $K$ the complete set. ${ }^{16}$ Since $\bar{T}$ is not recursively enumerable, $\bar{K}$, which consists of the positive integers corresponding to the elements of $\bar{T}$, is not recursively enumerable. Now let $B$ be the basis of a recursively enumerable subset $\alpha$ of $\bar{K}$. The elements of $E$ corresponding to the members of $\alpha$ constitute, then, a recursively enumerable subset $F$ of $\bar{T}$. Find then the $\left(B_{v}, \nu\right)$ of $\bar{T}$ not in $F$, and, via $O^{\prime}$, the positive integer $n$ corresponding to $\left(B_{\nu}, \nu\right)$. This $n$ will then be an element of $\bar{K}$ not in $\alpha$.

Actually, we have no general method of telling when a basis $B$ defines a recursively enumerable subset of $\bar{K}$. Indeed, the above method will yield a unique positive integer $n$ for any basis $B$ of a recursively enumerable set $\alpha$ of positive integers. However, when $\alpha$ is a subset of $\bar{K}, n$ will also be in $\bar{K}$, but not in $\alpha$.

Furthermore, even the formal proof of this result merely gives an effective method for finding $n$, given $B$. But this method itself can be formalized, so that, as a result, $n$ is given as a "recursive function of $B$." This can mean that a recursive function $f(m)$ can be set up such that $n=f(m)$ where $B=B_{m}$. We now isolate this property of $K$ by setting up the

Definition. $A$ creative set $C$ is a recursively enumerable set of positive integers for which there exists a recursive function giving a unique

15 See Kleene's Theorem XIII in [12] for a mathematically stateable theorem approximating the generality of our informal generalization.

16 "A complete set" might be better. Just how to abstract from $K$ the property of completeness is not, at the moment, clear. By contrast, see "creative set" below. 
positive integer $n$ for each basis $B$ of a recursively enumerable set of positive integers $\alpha$ such that whenever $\alpha$ is a subset of $\bar{C}, n$ is also in $\bar{C}$, but not in $\alpha$.

Theorem. There exists a creative set; to wit, the complete set $K$.

Actually, the class of creative sets is infinite, and very rich indeed as shown by the following easily proved results. ${ }^{17}$ If $C$ is a creative set, and $E$ a recursively enumerable set of positive integers, then if $E$ contains $\bar{C}, C E$ is creative, if $\bar{C}$ contains $E, C+E$ is creative. Results of $\$ 1$ enable us actually to construct creative sets according to the first method by using $E$ 's which are the complements of recursive subsets of $C$. Results of the rest of this section lead to constructions using the second method.

It is convenient to talk as if the $n$ in the definition of a creative set were determined by the $\alpha$ thereof instead of by the basis $B$ of $\alpha$. Clearly every creative set $C$ is a recursively enumerable set which is not recursive. For were $\bar{C}$ recursively enumerable, there could be no $n$ in $\bar{C}$ not in the recursively enumerable subset $\bar{C}$ of $\bar{C}$. The decision problem of each creative set is therefore recursively unsolvable. On the other hand, the complement $\bar{C}$ of any creative set $C$ contains an infinite recursively enumerable set. Recall that every finite set is recursive, and hence recursively enumerable. With, then, $\alpha$ of the definition of creative set as the null set, find the $n=n_{1}$ of $\bar{C}$ "not in $\alpha$." With $\alpha$ the unit set having $n_{1}$ as sole member, $n=n_{2}$ will be in $\bar{C}$, and distinct from $n_{1}$. With $\alpha$ consisting of $n_{1}$ and $n_{2}, n=n_{3}$ will be in $\bar{C}$, and distinct from $n_{1}$ and $n_{2}$, and so on. The set of positive integers $n_{1}, n_{2}$, $n_{3}, \cdots$ is then an infinite generated, and hence recursively enumerable, subset of $\bar{C}$.

Actually, with this subset of $\bar{C}$ as $\alpha$, a new element $n_{\omega}$ of $\bar{C}$ is obtained, and so on into the constructive transfinite. But this process is essentially creative. For any mechanical process could only yield $n$ 's forming a generated, and hence recursively enumerable, subset $\alpha$ of $\bar{C}$, and hence could be transcended by finding that $n$ of $\bar{C}$ not in $\alpha$.

4. One-one reducibility, to $K$; many-one reducibility. Let $S_{1}$ and $S_{2}$ be any two sets of positive integers. One of the simplest ways in which the decision problem of $S_{1}$ would be reduced to the decision problem of $S_{2}$ would arise if we had an effective method which would determine for each positive integer $n$ a positive integer $m$ such that $n$ is, or is not, in $S_{1}$ according as $m$ is, or is not, in $S_{2}$. For if we could

${ }^{17}$ Of course, all sets abstractly the same as a given creative set, in the sense of $\$ 1$, are creative. Likewise for our later simple and hyper-simple sets. 
somehow determine whether $m$ is, or is not, in $S_{2}$, we would determine $n$ to be, or not be, in $S_{1}$ correspondingly. If "effective method" be replaced by "recursive method," we shall say, briefly, that $S_{1}$ is then many-one reducible to $S_{2}$. If, furthermore, different $n$ 's always lead to different $m$ 's, we shall say that $S_{1}$ is one-one reducible to $S_{2}{ }^{18}$ "Recursive method" here can mean that $m=f(n)$, where $f(n)$ is a recursive function.

THEOREM. The decision problem of every recursively enumerable set of positive integers is one-one reducible to the decision problem of the complete set $K$.

For let $B^{\prime}$ be a basis of any one recursively enumerable set $S^{\prime}$. The effective one-one correspondence between all $(B, n)$ 's and all positive integers yielded by the effective enumeration $O^{\prime}$ of $E$, the set of all $(B, n)$ 's, then yields a unique positive integer $m$ for each $\left(B^{\prime}, n\right), B^{\prime}$ fixed, and thus a unique $m$ for each $n$, different $n$ 's yielding different $m$ 's. Now $n$ is, or is not, in $S^{\prime}$ according as $\left(B^{\prime}, n\right)$ is in $T$, or $\bar{T}$, and hence according as $m$ is in $K$, or $\bar{K}$, whence our result.

Since $K$ itself is recursively enumerable, we may say that for recursively enumerable sets of positive integers with recursively unsolvable decision problems there is a highest degree of unsolvability relative to one-one reducibility, namely, that of $K$. Actually, one-one reducibility is a special case of all the more general types of reducibility later introduced, and, though the proof of this is still in the informal stage, these latter are special cases of general recursive, that is, Turing reducibility. The same result then obtains relative to these special types of reducibility and, more significantly, for reducibility in the general sense. ${ }^{10}$

We have thus far explicitly obtained two recursively enumerable sets with recursively unsolvable decision problems, the $U$ of our first section, and $K$. We may note that a certain necessary and sufficient condition for the many-one reducibility of $K$ to a recursively enumerable set, the proof of which is still in the informal stage, has as an immediate consequence that $K$ is many-one reducible to $U$. It would then follow that $K$ and $U$ are of the same degree of unsolvability relative to many-one reducibility.

18 The resulting one-to-one correspondence is then between $S_{1}+\bar{S}_{1}$ and a subset, recursively enumerable indeed, of $S_{2}+\bar{S}_{2}$. Of course, both $S_{1}+\bar{S}_{1}$ and $S_{2}+\bar{S}_{2}$ constitute the set of all positive integers.

${ }^{19}$ It seems rather obvious that $K$ and the problem of Church [1] are each at least many-one reducible to the other; likewise for the problem of [1] and of [2, 3]. Had we verified this in detail, we would have called this highest degree of unsolvability of decisions problems of recursively enumerable sets the Church degree of unsolvability. 
5. Simple sets. It is readily proved that the necessary and sufficient condition that every recursive set be one-one reducible to a given recursively enumerable set of positive integers $S$ is that $S$ is infinite, and $\bar{S}$ contains an infinite recursively enumerable set. We are thus led to ask if there exist sets satisfying the following

Definition. A simple set is a recursively enumerable set of positive integers whose complement, though infinite, contains no infinite recursively enumerable set.

We now prove the

THEOREM. There exists a simple set.

Recall the set $T$ of all couples $(B, n)$ such that positive integer $n$ is in the recursively enumerable set of positive integers determined by basis $B$. Since $T$ is recursively enumerable, we can set up an effective enumeration

$$
O^{\prime \prime}: \quad\left(B_{i_{1}}, n_{1}\right),\left(B_{i_{2}}, n_{2}\right),\left(B_{i_{8}}, n_{3}\right), \cdots
$$

of its members. The subscript of each $B$ is its subscript in the effective enumeration $O: B_{1}, B_{2}, B_{3}, \cdots$ of all distinct $B$ 's. Now the complement of a set containing no infinite recursively enumerable set is equivalent to the set itself having an element in common with each infinite recursively enumerable set. Generate then the distinct bases $B_{1}, B_{2}, B_{3}, \cdots$, and as a $B_{i}$ is generated, regenerate the sequence $O^{\prime \prime}$ of $(B, n)$ 's in $T$, and the first time, if ever, $B$ is $B_{i}$, and $n$ is greater than $2 i$, place $n$ in a set $S$. The resulting set $S$ is then a generated, and hence recursively enumerable, set of positive integers. We proceed to prove it simple.

If $S^{\prime}$ is an infinite recursively enumerable set of positive integers, it will be determined by some basis $B_{i}$, and will have some element $m$ greater than $2 i$. Since $\left(B_{i}, m\right)$, being then in $T$, will appear in $O^{\prime \prime}$, our construction will place $m$ in $S$, if some earlier $\left(B_{i}, n\right)$ of $O^{\prime \prime}$ has not already contributed an element of $S^{\prime}$ to $S$. That is, $S$ has an element in common with each infinite recursively enumerable $S^{\prime}$. As for $\bar{S}$ being infinite, note that each $B_{i}$ contributes at most one element to $S$. The first $n B$ 's in $O$ therefore contribute at most $n$ elements to $S$. Each $B_{i}$ with $i \geqq n+1$ can only contribute to $S$ an element greater than $2 n+2$. Of the first $2 n+2$ positive integers, at most $n$ are therefore in $S$, and hence at least $n+2$ are in the consequently infinite $\bar{S} .{ }^{20}$

${ }^{20} n>i$ can replace $n>2 i$ in the above construction, but the proof will then depend on there being an infinite number of bases defining the null set. 
Having one simple set, the method of our succeeding $\$ 8$ can be modified to yield a rich infinite class of simple sets. Clearly, every simple set $S$ is a recursively enumerable set that is not recursive. For were $S$ recursive, $\bar{S}$ would be an infinite recursively enumerable subset of $\bar{S}$. The decision problem of each simple set is therefore recursively unsolvable. We thus have obtained two infinite mutually exclusive classes of recursively enumerable sets with recursively unsolvable decision problems, the class of creative sets, and the class of simple sets. They are poles apart in that the complements of creative sets have a creative infinity of infinite recursively enumerable subsets, those of simple sets, not one.

In passing, we may note that every recursively enumerable set of positive integers $S$ with recursively unsolvable decision problem leads to an incompleteness theorem for symbolic logics relative to the class of propositions $n \in S, n$ an arbitrary positive integer. Creative sets $S$ are then exactly those recursively enumerable sets of this type each of which admits a universal extendibility theorem as well, simple sets $S$ those for which, given $S$, each logic can prove the falsity of but a finite number of the infinite set of false propositions $n \in S$.

It is readily seen that no creative set $C$ can be one-one reducible to a simple set $S$. For under such a reduction, each infinite recursively enumerable subset of $\bar{C}$, proved above to exist, would be transformed into an infinite recursively enumerable subset of $\bar{S}$, contradicting the simplicity of $S$. Simple sets thus offer themselves as candidates for recursively enumerable sets with decision problems of lower degree of unsolvability than that of the complete set $K$. Even for many-one reducibility the situation is no longer immediately obvious; for an infinite recursively enumerable subset of $\bar{C}$ could thus be transformed into a finite subset of $\bar{S}$, the complement of simple $S$, without contradiction. However we can actually go much further than that.

6. Reducibility by truth-tables. If $S_{1}$ is many-one reducible to $S_{2}$, positive integer $n$ being, or not being, in $S_{1}$ may be said to be determined by its correspondent $m$ being, or not being, in $S_{2}$ in accordance with the truth-table

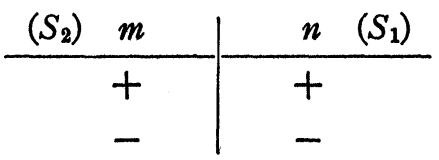

Here, the two signs,+- under $m$ represent the two possibilities $m$ is in $S_{2}, m$ is not in $S_{2}$, respectively. And by the sign under $n$ in the 
same horizontal row as the corresponding sign under $m$ the table in the same language tells whether $n$ correspondingly is $(+)$, or is not $(-)$, in $S_{1}$. The table then says that when $m$ is in $S_{2}, n$ is in $S_{1}$, when $m$ is not in $S_{2}, n$ is not in $S_{1}$, as required by many-one reducibility. Now there are altogether four ways in which $n$ being, or not being, in $S_{1}$ can be made to depend solely on $m$ being, or not being, in $S_{2}$, the signs under $n$ being,+- as above; or,$++;-,-;-,+$. If then we have an effective method which for each positive integer $n$ will not only determine a unique corresponding positive integer $m$, but also one of these four "first order" truth-tables, and if in each case the table is such that for the correct statement of membership or non-membership of $m$ in $S_{2}$, it gives the correct statement of membership or non-membership of $n$ in $S_{1}$, then the decision problem of $S_{1}$ will thus be reduced to the decision problem of $S_{2}$. For here also, given $n$, if we could somehow determine whether $m$ is, or is not, in $S_{2}$, we could thereby determine which row of the corresponding table correctly describes the membership or non-membership of $m$ in $S_{2}$, and from that row correctly determine whether $n$ is, or is not, in $S_{1}$.

More generally, let there be an effective method which for each positive integer $n$ determines a finite sequence of positive integers $m_{1}, m_{2}, \cdots, m_{\nu}, \nu$ as well as the $m$ 's depending on $n$. Let that method correspondingly determine for each $n$ a " $\nu$ th order" truth-table of the form

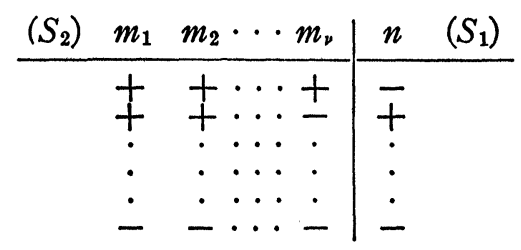

Each horizontal row, to the left of the vertical bar, specifies one of the $2^{\nu}$ possible ways in which the $\nu m_{i}$ 's may, or may not, be in $S_{2}$, to the right of the bar correspondingly commits itself to one of the statements $n$ is in $S_{1}, n$ is not in $S_{1}$. If then for each $n$ that row of the corresponding table which gives the correct statements for the $m$ 's being or not being in $S_{2}$ also gives the correct statement regarding the membership or non-membership of $n$ in $S_{1}$, the decision problem of $S_{1}$ is again thereby reduced to the decision problem of $S_{2}$.

If such a situation obtains with "effective method" replaced by "recursive method," we shall say that $S_{1}$ is reducible to $S_{2}$ by truthtables. "Recursive method" here can mean that a suitable Gödel representation of the couple consisting of the sequence $m_{1}, m_{2}, \cdots$, 
$m_{\nu}$ and the truth-table of order $\nu$ is a recursive function of $n$. If the orders of the truth-tables arising in such a reduction are bounded, we shall say that $S_{1}$ is reducible to $S_{2}$ by bounded truth-tables. Since there are $2^{2}$ distinct truth-tables of order $\nu$, reducibility by bounded truth-tables is equivalent to reducibility by truth-tables in which but a finite number of distinct tables arise.

7. Non-reducibility of creative sets to simple sets by bounded truth-tables. Let us suppose that creative set $C$ is reducible to simple set $S$ by bounded truth-tables. Let $T_{1}, T_{2}, \cdots, T_{\mathrm{k}}$ be the finite set of distinct truth-tables entering into such a reduction. That reduction then effectively determines for each positive integer $n$ a finite sequence of positive integers $m_{1}, m_{2}, \cdots, m_{v}$, and a unique $T_{i}, 1 \leqq i \leqq \kappa$.

The gist of our reductio-ad-absurdam proof consists in showing that under the assumed reduction we can obtain for each natural number $p$ a sequence of $m$ 's at least $p$ of which are in $S$. We then immediately have our desired contradiction. For in each case $p \leqq \nu$. The finite set of $\nu$ 's, the orders of the $T_{i}$ 's, being bounded, $p$ cannot then be arbitrarily large as stated.

More precisely we prove by mathematical induction that under the assumed reduction the following would be true. For each natural number $p$ an effective process $\Pi_{p}$ can be set up which will determine for each recursively enumerable subset $\alpha$ of $\bar{C}$ an element $n$ of $\bar{C}$ not in $\alpha$, and which for the corresponding $m_{1}, m_{2}, \cdots, m_{\nu}$ and $T_{i}$ yielded by the assumed reduction will correctly designate $p$ of these m's as belonging to $S$. The mode of designation may be assumed to be by specifying the sequence of subscripts, $i_{1}, i_{2}, \cdots, i_{p}$, of the $m$ 's to be designated, with say $i_{1}<i_{2}<\cdots<i_{p}$. With the assumed reduction adjoined to this process, $\Pi_{p}$ then determines for each $\alpha$ in question the quadruplet $\left(n, M, T_{i}, I\right), M$ being the sequence of $m$ 's, $I$ the sequence of subscripts of the $p$ designated $m$ 's.

For $p=0, \Pi_{p}$ is immediately given by the creative character of $C$. For that immediately gives us for each recursively enumerable subset $\alpha$ of $\bar{C}$ a definite element $n$ of $\bar{C}$ not in $\alpha$. The assumed reduction yields the corresponding $M$ and $T_{i}$; and with no members of $M$ designated as being in $S, I$ is the null sequence.

Inductively, assume that we have the process $\Pi_{p}$ for $p=k$. Let $\alpha$ be any given recursively enumerable subset of $\bar{C}$, and let $\left(n^{\prime}, M^{\prime}, T_{i^{\prime}}, I^{\prime}\right)$ be the corresponding quadruplet yielded by $\Pi_{k}$. Now suppose $n$ is a positive integer for which the assumed reduction yields the same table $T_{i^{\prime}}$ as it did for $n^{\prime}$, and a sequence of $m^{\prime} s, M$, consequently of the same length as $M^{\prime}$, having the following property. For each un- 
designated element of $M^{\prime}$, the correspondingly placed element of $M$ is identical with that of $M^{\prime}$; for each element of $M^{\prime}$ designated as being in $S$, the corresponding element of $M$ is also in $S$. Such an $n$ must then be in $\bar{C}$ along with $n^{\prime}$. For that row of $T_{i}$, which correctly tells of the $m$ 's of $M^{\prime}$ whether they are, or are not, in $S$ will also be the correct row for $M$. And since in the former case that row must say that $n^{\prime}$ is in $\bar{C}$, in the latter case it will say that $n$ is in $\bar{C}$, and correctly so. We proceed to show how all such $n$ 's may be generated.

We first show how to generate all $M$ 's obtainable from $M^{\prime}$ by replacing the designated elements of $M^{\prime}$ by arbitrary elements of $S$. For any one such $M$, the replacing elements, being finite in number, will be among the first $N$ elements, for some positive integer $N$, of a given recursive enumeration of $S$. Generate then the positive integers $1,2,3, \cdots$, and as a positive integer $N$ is generated, generate the first $N$ elements of the given recursive enumeration of $S$. For each $N$ place in a set $\beta$ the at most $N^{k}$ sequences $M$ that can be obtained from $M^{\prime}$ by replacing the designated elements of $M^{\prime}$ by elements chosen from the first $N$ elements of $S$. The generated set of sequences $\beta$ then consists of all $M$ 's obtainable from $M^{\prime}$ by replacing the designated elements of $M^{\prime}$ by arbitrary elements of $S$.

The $n$ 's we wish to generate are then those positive integers for which the assumed reduction yields the table $T_{i^{\prime}}$ and a sequence of $m$ 's, $M$, such that $M$ is a member of $\beta$. Generate then the elements of $\beta$. As an element $M$ of $\beta$ is generated, generate the positive integers $1,2,3, \cdots$, and as a positive integer $n$ is generated, find the corresponding sequence of $m$ 's and table yielded by the reduction of $C$ to $S$. If then that sequence of $m$ 's is $M$, and the table is $T_{i^{\prime}}$, add $n$ to the given set $\alpha$. As seen above, each such $n$ will be in $\bar{C}$. Hence the resulting generated, and hence recursively enumerable, set $\alpha^{\prime}$ is a subset of $\bar{C}$ containing $\alpha$. Our reason for thus adding the desired $n$ 's to $\alpha$ instead of just forming the class thereof is that the iterative process we are about to set up requires a cumulative effect.

As a result of our hypothesis and construction we thus have a derived process $\mathbb{I}_{k}^{\prime}$ which for every recursively enumerable subset $\alpha$ of $\bar{C}$ yields a definite recursively enumerable subset $\alpha^{\prime}$ of $\bar{C}$ containing $\alpha$. Starting with $\alpha$, we may then iterate the process $\Pi_{\mathbf{k}}^{\prime}$ to obtain the infinite sequence $A: \alpha_{1}, \alpha_{2}, \alpha_{3}, \cdots$, where $\alpha_{1}=\alpha, \alpha_{n+1}=\left(\alpha_{n}\right)^{\prime}$. Each member of $A$ is thus a recursively enumerable subset of $\bar{C}$, and contained in the next member of $A$. By applying the original process $\Pi_{k}$ to the members of $A$ we correspondingly obtain the infinite sequence $\Sigma: \sigma_{1}, \sigma_{2}, \sigma_{3}, \cdots$, where $\sigma_{j}$ is the quadruplet $\left(n^{(j)}, M^{(j)}, T_{i}^{(j)}\right.$, $\left.I^{(j)}\right)$ yielded by $\Pi_{k}$ for $\alpha_{j}$. We then observe the following. If for $j_{1} \neq j_{2}$ 
the $T$ 's of $\sigma_{j_{1}}$ and $\sigma_{j_{2}}$ are the same, and the $I$ 's are the same, then the sequences obtained from the $M$ 's by deleting the designated $m$ 's cannot be identical. For if they also were identical, then, with say $j_{1}<j_{2}$, $n^{\left(j_{2}\right)}$ would have been assigned to $\alpha^{\left(j_{1}+1\right)}$, whereas it actually is outside of $\alpha^{\left(j_{2}\right)}$ which contains $\alpha^{\left(j_{1}+1\right)}$. Hence, the infinite sequence $\Sigma^{\prime}$, obtained from $\Sigma$ by deleting from each $\sigma_{j}$ the integer $n^{(j)}$ and the designated $m$ 's of $M^{(j)}$, itself consists of distinct elements.

It follows that there are an infinite number of distinct undesignated $m$ 's appearing in $\Sigma$. Indeed, the distinct $T_{i}^{(j)}$ 's of $\Sigma$ are at most $\kappa$ in number. With $T_{i}^{(j)}$ fixed, the order $\nu^{(j)}$ of $T_{i}^{(j)}$ is fixed; and since $1 \leqq i_{1}^{(j)}<i_{2}^{(j)}<\cdots<i_{k}^{(j)} \leqq \nu^{(j)}$, the number of distinct $I$ 's is finite. Finally, with $T$ and $I$ fixed, were the total number of distinct undesignated $m$ 's finite, the number of distinct ways in which those $\nu^{(j)}-k$ undesignated $m$ 's could assume values would be finite. Hence $\Sigma^{\prime}$ would be finite, not infinite.

Now were each of this infinite set of undesignated $m$ 's in $\bar{S}$, we could regenerate the elements of $\Sigma$, and as an element $\sigma_{j}$ thereof is generated, place all of its undesignated $m$ 's in a set $\gamma$, and thus obtain an infinite generated, and hence recursively enumerable, subset of $\bar{S}$. As this contradicts the simplicity of $S$, it follows that at least one undesignated $m$ arising in $\Sigma$ is in $S$.

We can then find a unique such $m$, as well as a $\sigma$ in which it occurs, as follows. With $N=1,2,3, \cdots$, generate the first $N$ elements of the given recursive enumeration of $S$, and the first $N$ elements of $\Sigma$, and test the latter in order to see if any undesignated $m$ is among those first $N$ elements of $S$. If a particular undesignated $m$ of $\Sigma$ in $S$, proved above to exist, is the $L$ th member of $S$, and in the $K$ th member $\sigma_{K}$ of $\Sigma$, then an affirmative answer to the above test will certainly be obtained for $N=\max (L, K)$. Find then the first $N$ for which an affirmative answer is obtained, and let $(m, M, T, I)$ be the first $\sigma$ to yield the affirmative answer for this $N, m_{i^{\prime}}$ the first undesignated $m$ of $M$ thus found to be in $S$. We can then add $m_{i^{\prime}}$ to the designated $m$ 's of $M$, thus obtaining a quadruplet $\left(n, M, T_{i}, I_{1}\right)$, where $I_{1}$ designates $(k+1)$ of the $m$ 's of $M$ as being in $S$, and where $n$ is certainly a member of $\bar{C}$ not in the originally given $\alpha$. But the whole process leading up to $\left(n, M, T_{i}, I_{1}\right)$ is determined by that $\alpha$. It is therefore the desired process $\Pi_{p}$ for $p=k+1$.

Under the assumed reduction of $C$ to $S, \Pi_{p}$ would therefore exist for every natural number $p$. With $\alpha$ say the null set, we would thus obtain for every natural number $p$ a quadruplet $\left(n_{p}, M_{p}, T_{i_{p}}, I_{p}\right)$ such that $p$ of the members of the sequence $M_{p}$ are in $S$. Yet the total length of $M_{p}$ is the order of $T_{i_{p}}$, and hence bounded. Hence the 
TheOREM. No creative set is reducible to a simple set by bounded truth-tables.

We recall that every recursively enumerable set of positive integers is one-one reducible to the creative set $K$, the complete set. Hence the

CoROLlaRy. Every simple set is of lower degree of unsolvability than the complete set $K$ relative to reducibility by bounded truth-tables.

8. Counter-example for unbounded truth-tables. We recall that for the particular simple set $S$ constructed in $\$ 5$, of the first $2 m+2$ positive integers at most $m$ were in $S, m$ being any positive integer. Hence, of the $m+1$ integers $m+2, m+3, \cdots, 2 m+2$, at least one is in $\bar{S}$. By setting $m=2^{n}-1$, with $n=1,2,3, \cdots$, we can effectively generate the infinite sequence of mutually exclusive finite sequences

$$
\sigma: \quad(3,4),(5,6,7,8), \cdots,\left(2^{n}+1,2^{n}+2, \cdots, 2^{n+1}\right), \cdots
$$

such that each sequence in $\sigma$ has at least one member thereof in $\bar{S}$. An effective one-one correspondence between the positive integers 1,2 , $3, \cdots$ and the elements of $\sigma$ is then obtained by making the positive integer $n$ correspond to the sequence $\left(2^{n}+1,2^{n}+2, \cdots, 2^{n+1}\right)$ constituting the $n$th element of $\sigma$.

Given a creative set $C$, regenerate the elements of $S$, placing each in a set $S_{1}$. Furthermore, regenerate the elements of $C$, and as an element $n$ thereof is generated, place all of the positive integers in the $n$th sequence of $\sigma$ in $S_{1}$. The resulting set $S_{1}$ is a generated, and hence recursively enumerable, set of positive integers. Since $S_{1}$ contains $S, \bar{S}$ contains $\bar{S}_{1}$. As $S$ is simple, $\bar{S}$, and hence $\bar{S}_{1}$, does not have an infinite recursively enumerable subset. Moreover, $\bar{S}_{1}$ is also infinite. For $\bar{C}$ is infinite. And, for each element of $\bar{C}$, the corresponding sequence in $\sigma$ has only those of its members that are already in $S$ also in $S_{1}$, and hence at least one element in $\vec{S}_{1}$. Hence, $S_{1}$ is simple.

Likewise we see that a positive integer $n$ is in $C$, or $\bar{C}$, according as all of the integers in the $n$th sequence of $\sigma$ are in $S_{1}$, or at least one is in $\bar{S}_{1}$. If then we make correspond to each positive integer $n$ the sequence of $2^{n}$ positive integers $\left(2^{n}+1,2^{n}+2, \cdots, 2^{n+1}\right)$, and the truth-table of order $2^{n}$ in which the sign under $n$ is + in that row in which the signs under the $2^{n}$ " $m$ 's" are all + , and in every other row the sign under $n$ is -, we have a reduction of $C$ to $S_{1}$ by truth-tables. Hence the

ThEOREM. For each creative set $C$ a simple set $S$ can be constructed such that $C$ is reducible to $S$ by unbounded truth-tables. 
Corollary. A simple set $S$ can be constructed which is of the same degree of unsolvability as the complete set $K$ relative to reducibility by truth-tables unrestricted.

Simple sets as such do not therefore give us the absolutely lower degree of unsolvability than that of $K$ we are seeking.

9. Hyper-simple sets. The counter-example of the last section suggests that we seek a set satisfying the following

Definition. $A$ hyper-simple set $H$ is a recursively enumerable set of positive integers whose complement $\bar{H}$ is infinite, while there is no infinite recursively enumerable set of mutually exclusive finite sequences of positive integers such that each sequence has at least one member thereof in $\bar{H} .^{21}$

In this definition we may use the original Gödel method for representing a finite sequence of positive integers $m_{1}, m_{2}, \cdots, m_{\text {, }}$ by the single positive integer $2^{m_{1}} 3^{m_{2}^{*}} \cdots p^{m_{\nu}}$, where $2,3, \cdots, p_{\nu}$ are the first $\nu$ primes in order of magnitude. A set of finite sequences of positive integers is then recursively enumerable if the set of Gödel representations of those sequences is recursively enumerable.

Theorem. A hyper-simple set exists.

Our intuitive argument must again draw upon the formal development to the effect that each recursively enumerable set of finite sequences of positive integers will be determined by a "basis" $B^{*}$, and that all such bases can be generated in a single infinite sequence of distinct bases

$$
O^{*}: \quad B_{1}^{*}, B_{2}^{*}, B_{3}^{*}, \cdots
$$

As in $\$ 2$, generate the elements of $O^{*}$, and as an element $B^{*}$ is generated, set up the process for generating the set of sequences determined by $B^{*}$, and as a sequence $s$ is thus generated, write down the couple $\left(B^{*}, s\right)$. The resulting set of couples is then a generated set, and can indeed be effectively ordered in a sequence of distinct couples

$$
O_{1}^{*}: \quad\left(B_{i_{1}}^{*}, s_{1}\right),\left(B_{i_{2}}^{*}, s_{2}\right),\left(B_{i_{3}}^{*}, s_{3}\right), \cdots
$$

${ }^{21}$ Mutually exclusive sequences here mean no element of one sequence is an element of another. Curry suggests that "hyper-simple" is linguistically objectionable, and should be replaced by "super-simple." But we would not then know what to use in place of the letter $H$. 
$O_{1}{ }^{*}$ then consists of all distinct couples $\left(B^{*}, s\right)$ such that finite sequence $s$ is a member of the recursively enumerable set of finite sequences of positive integers determined by basis $B^{*}$.

Now the condition that no infinite recursively enumerable set of mutually exclusive finite sequences of positive integers has the property that each sequence has at least one positive integer thereof in $\bar{H}$ is equivalent to each such set of sequences having at least one sequence all of whose members are in $H$. Our method of constructing the desired hyper-simple set $H$ will then consist in placing in $H$ for certain $B^{* \prime}$ s in $O^{*}$ all of the positive integers in a sequence in the set of sequences determined by $B^{*}$. For purposes of presentation we shall call each such basis $B^{*}$ a contributing basis, while every $B^{*}$ determining an infinite recursively enumerable set of mutually exclusive sequences will be called a relevant basis. Set $H$, if recursively enumerable, will then be hyper-simple if each relevant basis is a contributing basis, and $\bar{H}$ is infinite.

If $B^{*}$ is a relevant basis, then among the infinite number of mutually exclusive sequences generated by $B^{*}$ there must be a sequence each of whose elements exceeds an arbitrarily given positive integer $N$. For did every sequence generated by $B^{*}$ have as element one of the integers $1,2, \cdots, N$, for any $N+1$ of these sequences at least two would have one of these integers in common. We shall then generate $H$ by regenerating sequence $O_{1}{ }^{*}$, and, as an element $\left(B_{i_{n}}^{*}, s_{n}\right)$ thereof is generated, we shall place all the elements of $s_{n}$ in $H$ if $B_{i_{n}}^{*}$ has not thus been made a contributing basis earlier in the process, while the elements of $s_{n}$ are all greater than a certain positive integer $N_{n}$, about to be determined; otherwise none. Inductively, assume $N_{m}$ to have been determined for $1 \leqq m<n$, and thus the entire process up to the time $\left(B_{i_{n}}^{*}, s_{n}\right)$ was brought up for consideration. Let $B_{j_{1}}^{*}, B_{j_{2}}^{*}, \cdots, B_{j_{\nu}}^{*}$ be the bases that have thus far contributed to $H$, and in the order in which they became contributing bases. These bases are then distinct, and hence their subscripts, which give their position in the sequence $O^{*}$ of all distinct bases, are distinct. Let $k_{1}, k_{2}, \cdots, k_{\nu}$ be the largest integer placed in $H$ by the first contributing basis, by the first two, ..., by the first $\nu$. The result being cumulative, $k_{1} \leqq k_{2} \leqq \cdots \cdots k_{\nu}$. The crux of our construction is to make $N_{n}$ depend not on the history of all these $\nu$ contributions to $H$, but only on that part of that history up to and including the last contribution, if any, made by a $B^{*}$ preceding $B_{i_{n}}^{*}$ in $O^{*}$. Specifically, if $B_{j_{\mu}}^{*}$ is the last of the above $\nu$ contributing bases preceding $B_{i_{n}}^{*}$ in $O^{*}$, that is, with $j_{\mu}<i_{n}, N_{n}$ is to be one more than the largest integer present in $H$ as a result of all the contributions made up to and in- 
cluding the contribution made by $B_{j_{\mu}}^{*}$. That is, $N_{n}=k_{\mu}+1$. Actually, if none of the $\nu$ contributing bases precede $B_{t_{n}}^{*}$ in $O^{*}$, no condition is to be placed on $s_{n}$, and all of its elements are placed in $H$ so long as $B_{i_{n}}^{*}$ is distinct from the $\nu$ contributing bases obtained thus far,

Furthermore, in our induction assume that we have been able to keep a record of the sequence $B_{j_{1}}^{*}, B_{j_{2}}^{*}, \cdots, B_{j_{v}}^{*}$, of $k_{1}, k_{2}, \cdots, k_{v}$, and also of $j_{1}, j_{2}, \cdots, j_{\nu}$ up to the time $\left(B_{i_{n}}^{*}, s_{n}\right)$ was about to be generated. We then generate $\left(B_{i_{n}}^{*}, s_{n}\right)$, and by regenerating $O^{*}$ find the place of $B_{i_{n}}^{*}$ in $O^{*}$ thus determining the subscript $i_{n}$. Our criterion for determining whether, or no, the elements of $s_{n}$ are to be placed in $H$ then becomes effective. In the latter case, the record is unchanged as we generate $\left(B_{i_{n+1}}^{*}, s_{n+1}\right)$. In the former, $B_{t_{n}}^{*}$ is written into the record as $B_{j_{v+1}}^{*}, i_{n}$ as $j_{v+1}$ while we can write in for $k_{p+1}$ the maximum of $k_{\nu}$ and the largest integer in $s_{n}$. The entire process is thus effective at each stage, and $H$ is thus a generated, and hence recursively enumerable, set of positive integers. We proceed to prove it hyper-simple.

Let $B^{*}$ be any relevant basis. Of the finite number of bases preceding $B^{*}$ in $O^{*}$, but a finite number can be contributing bases. Let $B_{j_{\mu}}^{*}$ be the last of these contributing bases, if any, appearing in the sequence $B_{j_{1}}^{*}, B_{j_{2}}^{*}, B_{j_{3}}^{*}, \ldots$ of distinct contributing bases determined by the above generation of $H$. There will then be a sequence $s$ generated by $B^{*}$ each of whose elements is greater than $k_{\mu}+1$. When then $\left(B^{*}, s\right)$, a definite element of $O_{1}^{*}$, is generated in the course of generating $H, B^{*}$ will contribute each element of $s$ to $H$ unless it became a contributing basis earlier in the process. Hence, every relevant basis is a contributing basis.

It also follows, or is easily seen directly, that the number of contributing bases is infinite. Consider then the infinite sequence of contributing bases $B_{j_{1}}^{*}, B_{j_{2}}^{*}, B_{j_{3}}^{*}, \ldots$, the corresponding infinite sequence of subscripts $j_{1}, j_{2}, j_{3}, \cdots$, and the associated infinite sequence $k_{1}, k_{2}, k_{3}, \ldots$. Since the contributing bases are distinct, so are their subscripts. Hence, for each $j_{m}$, among the infinite set of $j$ 's following $j_{m}$ there is a unique least $j, j_{m^{\prime}}$. Consider then the resulting infinite sequence $j_{\lambda_{1}}, j_{\lambda_{2}}, j_{\lambda_{8}}, \ldots$, where $j_{\lambda_{1}}$ is the least $j$ in the whole infinite sequence of $j$ 's, while $\lambda_{2}=\left(\lambda_{1}\right)^{\prime}, \lambda_{3}=\left(\lambda_{2}\right)^{\prime}, \ldots$. Now $k_{\lambda_{n}}$ is the largest integer contributed to $H$ through the contributing basis with subscript $j_{\lambda_{n}}$. Since $j_{\lambda_{n}}$ is the smallest $j$ following $j_{\lambda_{n-1}}$ it is less than all succeeding $j$ 's. Hence $B^{*}$ with subscript $j_{\lambda_{n}}$ precedes in $O^{*}$ all bases following that $B^{*}$ in the above infinite sequence of contributing bases. Hence, each element added to $H$ by contributing bases thus following $B^{*}$ with subscript $j_{\lambda_{n}}$ must exceed $k_{\lambda_{n}}+1$. It fol- 
lows, on the one hand, that for each positive integer $n, k_{\lambda_{n}}+1$ is in $\bar{H}$. On the other hand, $k_{\lambda_{n+1}}$ itself exceeds $k_{\lambda_{n}}+1$ so that $k_{\lambda_{n+1}}+1>k_{\lambda_{n}}+1$. These members of $\bar{H}$ therefore constitute an infinite subset of the consequently infinite $\bar{H}$. Hence, $H$ is hyper-simple.

Clearly, every hyper-simple set $H$ is simple. For an infinite recursively enumerable subset of $\bar{H}$, as set of unit sequences, would contradict $H$ being hyper-simple. Our construction of $\S 6$, in view of $\S 8$, gives us, however, a simple set which is not hyper-simple. Hypersimple sets thus constitute a third class of recursively enumerable sets with recursively unsolvable decision problems-a class which is a proper subclass of the class of simple sets.

10. Non-reducibility of creative sets to hyper-simple sets by truthtables unrestricted. Let creative set $C$ be reducible by truth-tables to a recursively enumerable set of positive integers $H$. The given reduction will again determine for each positive integer $n$ a finite sequence of positive integers $m_{1}, m_{2}, \cdots, m_{\nu}$, and a truth-table $T$ of order $\nu$ such that that row of the table which correctly tells of the $m$ 's whether they are, or are not, in $H$ will correctly tell of $n$ whether it is, or is not, in $C$. Of course $\nu$ and $T$ as well as the $m$ 's depend on $n$, and the set of distinct $T$ 's now entering into our reduction may be infinite, and hence the set of distinct $\nu$ 's unbounded.

Let $l_{1}, l_{2}, \ldots, l_{\mu}$ be any given finite sequence of distinct positive integers. A particular hypothesis on the $l$ 's being, or not being, in $H$ may then be symbolized by a sequence of $\mu$ signs, each + or - , such as $+-\cdots+$, such that the $i$ th sign is + , or - , according as the hypothesis says that $l_{i}$ is in $H$, or $\bar{H}$, respectively. We shall speak of such a sequence of signs as a truth-assignment for the $l$ 's, the $i$ th sign in that sequence as the sign of $l_{i}$ in that truth-assignment. Of the $2^{\mu}$ possible truth-assignments for the $l$ 's, constituting a set $V_{1}$, one and only one correctly tells of each $l_{i}$ whether it is, or is not, in $H$. Every set $V$ of truth-assignments for the $l$ 's is then a subset of $V_{1}$, and will be called a possible set of truth-assignments if it includes this correct truth-assignment.

Let then $V$ be any given possible set of truth-assignments for the $l$ 's. Let $n$ be a positive integer with corresponding $m_{1}, m_{2}, \cdots, m_{\nu}$, $T$ yielded by the given reduction of $C$ to $H$ such that each $m$ not an $l$ is in $H$. The correct row of table $T$ must then have the following two properties. First, the sign under each $m$ not an $l$ must be + . Second, the signs under those $m$ 's which are $l$ 's must be the same as the signs of those integers in some one and the same truth-assignment for the $l$ 's in $V$, in fact, as in the correct truth assignment for the $l$ 's. Any row 
of $T$ having these two properties, given the $l$ 's, $m$ 's and $V$, will be called a relevant row of $T$. Since for our $n$ the correct row of $T$ is thus a relevant row, it follows that $n$ will surely be in $\bar{C}$ if for each relevant row of $T$ the sign under $n$ is -.

Generate then the positive integers $1,2,3, \ldots$, and as a positive integer $N$ is generated, generate the first $N$ members of a given recursive enumeration of $H$, and for each $n$, with $1 \leqq n \leqq N$, find the corresponding $m_{1}, m_{2}, \cdots, m_{\nu}, T$ yielded by the given reduction of $C$ to $H$. Of those $m$ 's, if any, which are not $l$ 's, see if each is one of those first $N$ members of $H$. If they all are, see if for each relevant row of $T$ the sign under $n$ is -. If that also is true, place $n$ in a set $\alpha_{V}$. Since each such $n$ must be in $\bar{C}$, as seen above, $\alpha_{V}$ is a subset of $\bar{C}$. And being a generated set, $\alpha_{V}$ is therefore a recursively enumerable subset of $\bar{C}$.

$C$ being creative, we can therefore find a definite positive integer $n^{\prime}$ in $\bar{C}$ but not in $\alpha_{V}$, and, by the given reduction, the corresponding $m_{1}^{\prime}, m_{2}^{\prime}, \cdots, m_{\nu^{\prime}}^{\prime}, T^{\prime}$. Let $p_{1}, p_{2}, \cdots, p_{\lambda}$ be those $m^{\prime \prime}$, if any, which are not $l$ 's. Now suppose that each $p$ is in $H$. Then for at least one relevant row of $T^{\prime}$ the sign under $n^{\prime}$ must be + . For otherwise, if $p_{i}$ is say the $k_{i}$ th element in the given recursive enumeration of $H, n^{\prime}$ would have been placed in $\alpha_{V}$ in the above generation thereof for $N=\max \left(k_{1}, k_{2}, \cdots, k_{\lambda}, n^{\prime}\right)$. Since $n^{\prime}$ is in $\bar{C}$, such a relevant row cannot be the correct row. But, with each $p$ in $H$, the signs in that row under $m$ 's that are not $l$ 's are correctly + . Hence the sign under at least one $m^{\prime}$ that is an $l$ must be incorrect. But, by our definition of a relevant row, the signs under all such $m^{\prime \prime}$ s are the same as the signs of those integers in at least one truth-assignment in $V$. Such a truth-assignment in $V$ cannot therefore be the correct truth-assignment for the $l$ 's, and hence may be deleted from $V$. Perform this deletion for all such truth-assignments in $V$, and for all such relevant rows of $T^{\prime}$, to obtain the set of truth-assignments $V^{\prime}$. Under our hypothesis that each $p$ is in $H, V^{\prime}$ will then be a proper subset of $V$, and yet a possible set of truth-assignments for the $l$ 's.

Actually, let $V$ be any given set of truth-assignments for the $l$ 's, possible or not. Each step of the above construction can then still be carried out, though the constructed entities need not now have all the properties they otherwise possess. ${ }^{22}$ In particular, the set of integers, possibly null, $p_{1}, p_{2}, \cdots, p_{\lambda}$ can be found, all different from any $l$. Likewise, whether the $p$ 's are, or are not, all in $H$, the subset $V^{\prime}$ of $V$ can be found. What we can say is that if $V$ is a possible set,

${ }^{22}$ Recall that in the definition of creative set, $\$ 3$, each $B$ determines an $n$, whether the $\alpha$ determined by $B$ is, or is not, a subset of $\bar{C}$. 
and if furthermore each $p$ is in $H$, then $V^{\prime}$ is a proper subset of $V$, and itself is also a possible set of truth-assignments for the $l$ 's.

For the given sequence of $l$ 's, start then with $V=V_{1}$, the possible set of all $2^{\mu}$ truth-assignments for the $l$ s, obtain the corresponding $p^{\prime} s, p_{1}^{\prime}, p_{2}^{\prime}, \cdots, p_{\lambda}^{\prime}$ and corresponding $V^{\prime}, V_{2}=\left(V_{1}\right)^{\prime}$. With $V=V_{2}$, likewise find $p_{1}^{\prime \prime}, p_{2}^{\prime \prime}, \cdots, p_{\lambda}^{\prime \prime}$, and $V_{8}=\left(V_{2}\right)^{\prime}$, and so on. Now each $V_{i+1}$ is a subset of $V_{i}$, while $V_{1}$ is but a finite set of $2^{\mu}$ members. Hence in at most $2^{\mu}$ steps we shall come across a $V_{k}$ such that either $V_{k+1}$ is identical with $V_{k}$, or is null. But if all the $p^{\prime \prime}$,,$p^{\prime \prime \prime}$ s, $\cdots, p^{(k)}$ 's were in $H, V_{1}$ being a possible set, $V_{2}, \cdots, V_{k}$ as well as $V_{k+1}$ would all be possible sets, each a proper subset of the preceding. $V_{k+1}$ could not then either be identical with $V_{k}$, or null. It follows that at least one of the $p_{i}^{(j)}$ 's with $1 \leqq j \leqq \kappa$ is in $\bar{H}$. Each $p_{i}^{(j)}$ is an integer that is not one of the $l$ 's. If then we take this finite set of $p_{i}^{(j)}$ 's and arrange them in a sequence of distinct elements in say order of magnitude, we obtain for our arbitrarily given sequence of distinct positive integers $l_{1}, l_{2}, \cdots, l_{\mu}$ a sequence of distinct positive integers $k_{1}, k_{2}, \cdots, k_{\nu}$ having no element in common with the former sequence, and having at least one element in $\bar{H}$.

Starting with the null sequence as the sequence of $l$ 's, we can thus find the sequence of $k^{\prime} s,\left(k_{1}^{\prime}, k_{2}^{\prime}, \cdots, k_{\nu^{\prime}}^{\prime}\right)$ of distinct positive integers at least one of which is in $\bar{H}$. Inductively, let us have thus generated the sequences $\left(k_{1}^{\prime}, k_{2}^{\prime}, \cdots, k_{\nu^{\prime}}^{\prime}\right), \cdots,\left(k_{1}^{(\mu)}, k_{2}^{(\mu)}, \cdots, k_{\nu(\mu)}^{(\mu)}\right)$, mutually exclusive, of distinct positive integers, each having at least one element in $\bar{H}$. With the single sequence $k_{1}^{\prime}, \cdots, k_{\nu}^{(\mu)}(\mu)$ as the sequence of $l$ 's, we can find the corresponding sequence of $k^{\prime} s,\left(k_{1}^{(\mu+1)}, k_{2}^{(\mu+1)}, \cdots\right.$, $\left.k_{\nu(\mu+1)}^{(\mu+1)}\right)$ of distinct positive integers with no element in common with any of the preceding sequences, and having at least one element in $\bar{H}$.

With creative $C$ reducible to recursively enumerable $H$ by truthtables we can thus obtain an infinite generated, and hence recursively enumerable, set of mutually exclusive finite sequences of positive integers each having an element in $\bar{H}$. The set $H$ is therefore not hyper-simple. Hence the

THEOREM. No creative set is reducible to a hyper-simple set by truthtables.

COROLLARY. Every hyper-simple set is of lower degree of unsolvability than the complete set $K$ relative to reducibility by truth-tables.

Despite this result, the brief discussion of Turing reducibility, still in the informal stage, entered into in the next section makes it dubious that hyper-simple sets as such will give us the desired absolutely 
lower degree of unsolvability than that of $K$. But, in the absence of a counter-example, they remain candidates for this position.

11. General (Turing) reducibility. The process envisaged in our concept of a generated set may be said to be polygenic. In a monogenic process act succeeds act in one time sequence. The intuitive picture is that of a machine grinding out act after act (Turing [24]) or a set of rules directing act after act (Post [18]). The actual formulations are in terms of "atomic acts," the first leading to a development proved by Turing [25] equivalent to those arising from general recursive function or $\lambda$-definability, and hence of the same degree of generality. In our intuitive discussion the acts may be "molecular."

An effective solution of the decision problem for a recursively enumerable set $S_{1}$ of positive integers may therefore be thought of as a machine, or set of rules, which, given any positive integer $n$, will set up a monogenic process terminating in the correct answer, "yes" or "no," to the question "is $n$ in $S_{1}$." Now suppose instead, says Turing [26] in effect, this situation obtains with the following modification. That at certain times the otherwise machine determined process raises the question is a certain positive integer in a given recursively enumerable set $S_{2}$ of positive integers, and that the machine is so constructed that were the correct answer to this question supplied on every occasion that arises, the process would automatically continue to its eventual correct conclusion. ${ }^{28}$ We could then say that the machine effectively reduces the decision problem of $S_{1}$ to the decision problem of $S_{2}$. Intuitively this should correspond to the most general concept of the reducibility of $S_{1}$ to $S_{2}$. For the very concept of the decision problem of $S_{2}$ merely involves the answering for an arbitrarily given single positive integer $m$ of the question is $m$ in $S_{2}$; and in finite time but a finite number of such questions can be asked. A corresponding formulation of "Turing reducibility" should then be the same degree of generality for effective reducibility as say general recursive function is for effective calculability. ${ }^{24}$

We may note that whereas in reducibility by truth-tables the posi-

${ }_{28}$ Turing picturesquely suggests access to an "oracle" which would supply the correct answer in each case. The "if" of mathematics is however more conducive to the development of a theory.

${ }^{24}$ A reading of McKinsey [16] suggested generalizing the reducibility of a recursively enumerable set $S$ to a recursively enumerable set $S^{\prime}$ to the reducibility of $S$ to a finite set of recursively enumerable sets $S_{1}, S_{2}, \cdots, S_{n}$. However, no absolute gain in generality is thus achieved, as a single recursively enumerable set $S^{\prime}$ can be constructed such that reducing $S$ to $\left(S_{1}, S_{2}, \ldots, S_{n}\right)$ is equivalent to reducing $S$ to $S^{\prime}$. Points of interest, however, do arise. 
tive integers $m$ of which we ask the questions "is $m$ in $S_{2}$ " are effectively determined, for given $n$, by the reducibility process, in Turing reducibility, except for the first such $m$, the very identity of the $m$ 's for which this question is to be asked depends, in general, on the correct answers having been given to these questions for all preceding $m$ 's. The mode of this dependence is, however, effective, hence we still have effective reducibility in the intuitive sense.

Let now creative set $C$ be Turing reducible to a recursively enumerable set $S$ of positive integers. We shall talk as if our intuitive discussion has already been formalized. Generate the positive integers $1,2,3, \cdots$, and as a positive integer $N$ is generated, for each $n$ with $1 \leqq n \leqq N$ proceed as follows. Set going the reducibility process of $C$ to $S$ for $n$. Each time a question of the form "is $m$ in $S$ " is met, see if $m$ is among the first $N$ integers in a given recursive enumeration of $S$. If it is, supply the answer "yes," thus enabling the reducibility process to continue. Finally, if under these circumstances the process terminates in a "no" for the initial question of $n$ being in $C$, place $n$ in a set $\alpha_{0}$. This $\alpha_{0}$ is then a recursively enumerable subset of $\bar{C}$ consisting of all members thereof for which the given Turing reduction of $C$ to $S$ leads only to questions of the form "is $m$ in $S$ " whose answer is "yes."

Find then $n_{0}$ of $\bar{C}$ not in $\alpha_{0}$, and set the reducibility process going for $n_{0}$. Now if at any time a wrong answer is supplied to a question "is $m$ in $S$," we can nevertheless expect our machine for reducing $C$ to $S$ either to effectively pick up the wrong answer and operate on it to give a next step in the process, or to cease operating. Generate then the positive integers $1,2,3, \cdots$, and as a positive integer $N$ is generated, generate the first $N$ members of the given recursive enumeration of $S$, and make the reducibility process for $n_{0}$ effective though perhaps incorrect as follows. Each time a question of the form "is $m$ in $S$ " is reached, see if $m$ is among the first $N$ members of $S$. If it is, answer the question "yes," and correctly so; if not, answer the question "no," whether that answer be correct or no. If now this pseudoreduction terminates in a "no," place the finite number of $m$ 's thus arising in a set $\beta_{n_{0}}$. Note that $\beta_{n_{0}}$ consists of all such $m$ 's for all such pseudo-reductions for the given $n_{0}$. Being a generated set of positive integers, $\beta_{n_{0}}$ is recursively enumerable.

Now let the correct, though possibly non-effective, reducibility process for $n_{0}$ involve the $\mu$ questions "is $m_{i}$ in $S$, , $i=1,2, \ldots, \mu$. Let $m_{i_{1}}, m_{i_{2}}, \cdots, m_{i_{y}}$ be those of these $m$ 's actually in $S$, and let them be the $n_{1}$ st, $n_{2}$ nd, $\cdots, n_{\nu}$ th members of the given recursive enumeration of $S$. If then $N \geqq M=\max \left(n_{1}, n_{2}, \cdots, n_{v}\right)$, or $M=1$ if 
$\nu=0$, the corresponding psuedo-reduction for $n$ becomes the correct reduction. For, inductively, if that be so through the time a question "is $m$ in $S$ " is raised, $m$ will be $m_{1}, m_{2}, \cdots$, or $m_{\mu}$, hence will, or will not, be in $S$ according as it is, or is not, one of the first $N$ members of $S$. The answer is then correctly given by that pseudo-reduction, which therefore continues to be correct through the raising of the next question. Finally, since $n_{0}$ is in $\bar{C}$, the correct reduction, now the pseudo-reduction, must terminate with a "no."

It follows that all $N$ 's with $N>M$ merely repeat the contribution to $\beta_{n_{0}}$ made by $N=M$, that is, of the integers $m_{1}, m_{2}, \cdots, m_{\mu}$. Since but a finite number of $m$ 's are contributed by $N$ 's with $N<M$, it follows that $\beta_{n_{0}}$ is a finite set. Finally, were each of the integers $m_{1}, m_{2}, \cdots, m_{\mu}$ in $S, n_{0}$ would be in $\alpha_{0}$. Hence, at least one member of $\beta_{n_{0}}$ is in $\bar{S}$.

Formally, we would thus obtain a basis for a finite recursively enumerable set of positive integers at least one of whose members is in $\bar{S}$. Instead of recursively enumerable sets of finite sequences of positive integers, we would thus be led to consider recursively enumerable sets of bases for finite recursively enumerable sets of positive integers. Though, in the last analysis, each sequence in the former case must be generated atom by atom, there will come a time for each sequence when the process will say "this sequence is completed." In the latter case, in general, we cannot have an effective method which, for each basis, will give a point in the ensuing process at which it can say all members of the finite set in question have already been obtained, even though, with the process made monogenic, there always is such a stage in the process.

This suggests, then, that we strengthen the condition of hypersimplicity still further by replacing "infinitive recursively enumerable set of mutually exclusive finite sequences of positive integers" in the definition of $\$ 9$ by "infinite recursively enumerable set of bases defining mutually exclusive finite recursively enumerable sets of positive integers." Whether such a "hyper-hyper-simple" set exists, or whether, if it exists, it will lead to a stronger non-reducibility result than that of the last section we do not know.

On the other hand, an equivalent definition of hyper-simple set is obtained if, for example, we replace the quoted phrase by "recursively enumerable set of finite sequences of positive integers having for each positive integer $n$ a member each of whose elements exceeds $n$." We now can say that with this as the definition of a hyper-simple set, the corresponding extension to a hyper-hyper-simple set cannot be made. For we prove the 
TheOREM. For any recursively enumerable set of positive integers $S$, with infinite $\bar{S}$, there exists a recursively enumerable set of bases defining finite recursively enumerable sets of positive integers, each set having at least one element in $\bar{S}$, and at least one set having each of its elements greater than an arbitrarily given positive integer $n$.

Briefly, with $n$ given, for each positive integer $N$, and each positive integer $m$, place all of the integers $n+1, n+2, \cdots, n+m$ in a set $\alpha_{n}$ if all, or all but the last, are among the first $N$ members of a given recursive enumeration of $S$. It is readily seen that $\alpha_{n}$ is a generated, and hence recursively enumerable, set of positive integers. A corresponding basis $B^{(n)}$ can actually be found, and the set of $B^{(n)}$ 's, $n=1,2,3, \cdots$, being a generated set, is therefore recursively enumerable. Moreover, if $\nu_{n}$ is the smallest integer in the infinite $\bar{S}$ greater than $n, \alpha_{n}$ will consist of exactly the integers $n+1, n+2, \cdots$, $\nu_{n}$, and hence will be finite, with indeed $\nu_{n}$ as the only element in $\vec{S}$, and with each element greater than $n$.

As a result we are left completely on the fence as to whether there exists a recursively enumerable set of positive integers of absolutely lower degree of unsolvability than the complete set $K$, or whether, indeed, all recursively enumerable sets of positive integers with recursively unsolvable decision problems are absolutely of the same degree of unsolvability. On the other hand, if this question can be answered, that answer would seem to be not far off, if not in time, then in the number of special results to be gotten on the way. ${ }^{25}$

Such then is the portion of "Recursive theory" we have thus far developed. In fixing our gaze in the one direction of answering the lower degree of unsolvability question, we have left unanswered many questions that stud even the short path we have traversed. Moreover, both our special, and the general Turing, definitions of reducibility are applicable to arbitrary decision problems whose questions in symbolic form are recursively enumerable, and indeed to problems with recursively enumerable set of questions whose answers belong to a recursively enumerable set. Thus, only partly leaving the field of decisions problems of recursively enumerable sets, work of Turing [26] suggests the question is the problem of determining of an arbitrary basis $B$ whether it generates a finite, or infinite, set of positive

${ }^{25}$ This is a matter of practical concern as well as of theoretical interest. For according as the second or first of the above alternatives holds will the method of reducing new decision problems to problems previously proved unsolvable be, or not be, the general method for proving the unsolvability of decision problem either of recursively enumerable sets of positive integers or of problems equivalent thereto. 
integers of absolutely higher degree of unsolvability than $K$. And if so, what is its relationship to that decision problem of absolutely higher degree of unsolvability than $K$ yielded by Turing's theorem.

Actually, the theory of recursive reducibility can be but one chapter in the theory of recursive unsolvability, and that, but one volume of the theory and applications of general recursive functions. Indeed, if general recursive function is the formal equivalent of effective calculability, its formulation may play a rôle in the history of combinatory mathematics second only to that of the formulation of the concept of natural number.

\section{BIBLIOGRAPHY}

1. Alonzo Church, An unsolvable problem of elementary number theory, Amer. J. Math. vol. 58 (1936) pp. 345-363.

2. - A note on the Entscheidungsproblem, Journal of Symbolic Logic vol. 1 (1936) pp. 40-41.

3. - Correction to a note on the Entscheidungsproblem, ibid. pp. 101-102.

4. - Combinatory logic as a semi-group, Preliminary report, Bull. Amer. Math. Soc. abstract 43-5-267.

5. - The constructive second number class, ibid. vol. 44 (1938) pp. 224-232.

6. - The calculi of lambda-conversion, Annals of Mathematics Studies, no. 6, Princeton University Press, 1941.

7. C. J. Ducasse, Symbols, signs, and signals, Journal of Symbolic Logic vol. 4 (1939) pp. 41-52.

8. Kurt Gödel, Über formal unentscheid̂bare Sätze der Principia Mathematica und verwandter Systeme I, Monatshefte für Mathematik und Physik vol. 38 (1931) pp. 173-198.

9. - On undecidable propositions of formal mathematical systems, mimeographed lecture notes, The Institute for Advanced Study, 1934.

10. David Hilbert, Mathematical problems. Lecture delivered before the International Congress of Mathematicians at Paris in 1900. English translation by Mary Winston Newsom, Bull. Amer. Math. Soc. vol. 8 (1901-1902) pp. 437-479.

11. David Hilbert and Paul Bernays, Grundlagen der Mathematik, vol. 2, Julius Springer, Berlin, 1939.

12. S. C. Kleene, General recursive functions of natural numbers, Math. Ann. vol. 112 (1936) pp. 727-742.

13. - On notation for ordinal numbers, Journal of Symbolic Logic vol. 3 (1938) pp. 150-155.

14. - Recursive predicates and quantifiers, Trans. Amer. Math. Soc. vol. 53 (1943) pp. 41-73.

15. C. I. Lewis, A survey of symbolic logic, Berkley, 1918, chap. $6, \S 3$.

16. J. C. C. McKinsey, The decision problem for some classes of sentences without quantifiers, Journal of Symbolic Logic vol. 8 (1943) pp. 61-76.

17. Rozsa Peter, Az axiomatikus modszer korlatai (The bounds of the axiomatic method), Review of, Journal of Symbolic Logic vol. 6 (1941) pp. 111.

18. Emil L. Post, Finite combinatory processes-formulation 1, ibid. vol. 1 (1936) pp. 103-105. 
19. - Formal reductions of the general combinatorial decision problem, Amer. J. Math. vol. 65 (1943) pp. 197-215.

20. J. B. Rosser, Extensions of some theorems of Gödel and Church, Journal of Symbolic Logic vol. 1 (1936) pp. 87-91.

21. - An informal exposition of proofs of Gödel's theorems and Church's theorem, ibid. vol. 4 (1939) pp. 53-60.

22. Th. Skolem, Einfacher beweis der unmöglichkeit eines allgemeinen losungsverfahrens fitr arithmetische probleme, Review of, Mathematical Reviews vol. 2 (1941) p. 210.

23. Alfred Tarski, On undecidable statements in enlarged systems of logic and the concept of truth, Journal of Symbolic Logic vol. 4 (1939) pp. 105-112.

24. A. M. Turing, On computable numbers, with an application to the Entscheidungsproblem, Proc. London Math. Soc. (2) vol. 42 (1937) pp. 230-265.

25. - Computability and $\lambda$-definability, Journal of Symbolic Logic vol. 2 (1937) pp. 153-163.

26. - Systems of logic based on ordinals, Proc. London Math. Soc. (2) vol. 45 (1939) pp. 161-228.

\section{The City College,} New York City. 